\title{
Apolipoprotein B/apolipoprotein A1 ratio and mortality among incident peritoneal dialysis patients
}

\author{
Xiaojiang Zhan, Yanbing Chen, Caixia Yan, Siyi Liu, Lijuan Deng, Yuting Yang, Panlin Qiu, Dan Pan, \\ Bingxiang Zeng and Qinkai Chen ${ }^{*}$
}

\begin{abstract}
Background: To investigate the association between the ratio of apolipoprotein B (apo B) / apolipoprotein A1 (apo A1) with all-cause mortality and cardiovascular events in peritoneal dialysis (PD) patients.

Methods: Eight hundred and sixty incident PD patients were enrolled from November 1, 2005, to February 28, 2017, and followed until May 31, 2017. Outcomes were all-cause mortality and cardiovascular events. Associations between the apo B/apo A1 ratio with all-cause mortality and cardiovascular events were evaluated using multivariable-adjusted Cox models.

Results: Of the 860 patients, the mean age was $49.9 \pm 14.5$ years, $57.6 \%$ were men, and $19.3 \%$ were diabetic patients. The median apo B/apo A1 ratio was 0.65 (range: 0.22-2.24). During a median follow-up period of 27 months (interquartile range, 13 - 41 months), 202 deaths, and 145 cardiovascular events were recorded. After adjustment for age, sex, body mass index, diabetes, cardiovascular disease, systolic blood pressure, total Kt/ $\mathrm{N}$, estimated glomerular filtration rate, hemoglobin level, neutrophil to lymphocyte ratio and albumin, triglyceride, and cholesterol, as well as the use of lipid-lowering agents, the highest apo B/apo A1 ratio tertile was significantly associated with a hazard ratio for all-cause mortality of 1.60 ( $95 \%$ Cl: 1.02 to $2.49, P=0.040$ ) and for cardiovascular events of 2.04 (95\% Cl: 1.21 to 3.44 , $P=0.008)$.
\end{abstract}

Conclusion: An increased apo B/apo A1 ratio was independently associated with all-cause mortality and cardiovascular events in PD patients.

Keywords: Apolipoprotein, Cardiovascular event, Cohort study, Mortality, Peritoneal dialysis

\section{Background}

Peritoneal dialysis (PD) patients have an increased risk of morbidity and mortality associated with cardiovascular disease (CVD), which is, at least in part, due to lipid abnormalities, typically called uremic dyslipidemia [1]. Patients typically present with a decrease of the highdensity lipoprotein cholesterol (HDL-C), and an increase of triglyceride (TG) and lipoprotein (a) [Lp (a)], with relatively normal or even lower levels of low-density lipoprotein cholesterol (LDL-C) [2].

LDL-C is widely recognized as the major atherogenic lipoprotein and is considered the primary therapeutic

\footnotetext{
* Correspondence: timmyclz@163.com

Department of Nephrology, The First Affiliated Hospital of Nanchang

University, 17\# yongwai street, Nanchang 330006, Jiangxi, China
}

target for coronary heart disease [3]. Furthermore, multiple lines of evidence indicate that other TG-rich lipoproteins, including intermediate density lipoprotein (IDL) or very low-density lipoprotein (VLDL) carry atherogenic potential as well [4]. It has been reported that approximately $50 \%$ of patients with abnormalities of atherogenic lipoprotein had an increased risk for the development of CVD [5]. This led to the consideration of non-HDL cholesterol as another important target for hyperlipidemia treatment. It is well known that, lipidtransporting apolipoprotein is an essential structural component of the lipoprotein cholesterol [6], VLDL, IDL and LDL cholesterol can each carry only a single apolipoprotein B (apo B) [7] and this characteristic can be used to reliably reflected the number of atherogenic

(C) The Author(s). 2018 Open Access This article is distributed under the terms of the Creative Commons Attribution 4.0 International License (http://creativecommons.org/licenses/by/4.0/), which permits unrestricted use, distribution, and 
lipoproteins in the plasma. In contrast, apo A1 has a strong association with HDL levels and accounts for approximately $60-70 \%$ of the total apolipoproteins in HDL-C, the plasma content of apo A1 represents the total of antiatherogenic particles [8]. Therefore, the ratio of apo B to apo A1 (apo B/apo A1) reflects the balance of proatherogenic and antiatherogenic particles.

Recently, Sato et al. [9] indicated that a higher ratio of the apo B/apo A1 was associated with higher risk of allcause and CVD-related mortality in patients on hemodialysis (HD). However, the lipid profile of PD patients was different from HD patients. In particular the lipid profile is more atherogenic in nature with more altered dyslipidemia when compared with those in HD patients [10]. Moreover, the association between the apo $\mathrm{B} /$ apo A1 ratio and mortality in PD patients remains uncertain. Therefore, we hypothesized that a higher ratio of apo B/apo A1 is a critical parameter to predict the risk of cardiovascular events and all-cause mortality in PD patients. In this longitudinal cohort study, we assessed the associations between the apo B/apo A1 ratio with all-cause mortality and cardiovascular events in PD patients, and followed the patients for a median of 27 months at our PD center.

\section{Methods}

\section{Study population and data collection}

We studied all incident patients who used PD as their first renal replacement treatment modality and were followed up at the PD center of The First Affiliated Hospital, Nanchang University, Jiangxi, China from November 1, 2005, to February 28, 2017. Inclusion criteria were age $\geq 18$ years at the start of PD and survival for at least 90 days from the first PD therapy. The patients who were catheterized in other hospitals, transferred from permanent HD, or failed renal transplantation were excluded in this study. The study was conducted in compliance with the ethical principles of the Helsinki Declaration (https://jamanetwork.com/journals/jama/fullarticle/

1760318) and approved by the Human Ethics Committees of Nanchang University.

All patients were followed up until cessation of PD, death, or May 31, 2017. Baseline demographic data included age, sex, primary cause of end-stage renal disease (ESRD), and presence of diabetes and CVD. Clinical and biochemical data at the initiation of PD included body mass index, blood pressure, medication use, hemoglobin, serum albumin, serum creatinine, blood urea nitrogen, total cholesterol (CHOL), TG, HDL-C, LDL-C, Apo B, Apo A1, Lp (a). Immunoturbidimetric method was used to measure apo B and apo A1 concentrations. All baseline data were obtained during the first $1-3$ months of PD. Baseline residual renal function was assessed by eGFR using the Chronic Kidney Disease Epidemiology
Collaboration creatinine equation. Cardiovascular events were defined by the first occurrence of myocardial infarction, stroke, heart failure, hospitalization for unstable angina, peripheral vascular event, sudden death, death associated with a cardiovascular procedure, or death due to aneurysm dissection or rupture, fatal pulmonary embolism, or death due to other or unknown cardiovascular cause [11] after the onset of PD, and was determined by the PD follow-up panel composed of PD primary nurses and professors.

\section{Statistical analyses}

Patients with apo B/apo A1 ratio were classified into tertiles (Ts): $\mathrm{T} 1 \leq 0.545, \mathrm{~T} 2=0.545-0.769, \mathrm{~T} 3>0.769$. Participant characteristics were calculated by Ts of apo B/ apo A1. Results were expressed as frequencies and percentages for categorical variables, means and standard deviations (SDs) for normally distributed continuous variables, and medians and interquartile ranges for continuous variables not normally distributed. Chi-squared, one-way ANOVA, or Kruskal-Wallis tests were used to test for differences in categorical or continuous factors among different categories of apo B/apo A1. Survival times were estimated from Kaplan-Meier curves, and differences in survival probabilities among groups were assessed using the log-rank test. The associations between apo B/apo A1 ratio and all-cause mortality and cardiovascular events were examined in Cox proportional hazards models. The censored data included switching to $\mathrm{HD}$, renal transplantation, moving to another center, declining additional treatment, loss to follow-up, or still at our PD center on May 31, 2017. Unadjusted associations were first examined followed by adjustments for age and sex, diabetes and CVD, systolic blood pressure (SBP), body mass index (BMI), total urea clearances (tKt/V) and eGFR, hemoglobin, neutrophil to lymphocyte ratio (N/L), albumin. Next, TG, CHOL as well as lipid lowering agents were added to examine whether apo B/apo A1 ratio was independently associated with mortality and cardiovascular events. Covariates with $P<0.05$ in the univariate Cox analyses or thought to be clinical significant were chosen for multivariate Cox proportional hazards regression. The results were expressed as the hazard ratio (HR) and 95\% confidence interval $(95 \% \mathrm{CI})$. All descriptive and multivariate analyses were conducted using SPSS version 22.0 (SPSS, Inc., Chicago, IL). A value of $P<0.05$ was considered statistically significant.

\section{Results}

\section{Baseline patient characteristics}

A total of 1011 incident PD patients were recruited and monitored in our hospital. Thirty-four of the patients were excluded by our experimental criteria and listed as 
below: 3 subjects were under 18 years of age, 2 subjects were transferred from failed renal transplantation, 8 subjects were transferred from permanent HD, and 21 subjects were treated with PD for less than 3 months. The remaining 977 patients were enrolled. Of the 977 patients, 117 patients did not measure baseline levels of apolipoproteins. Finally, 860 patients were found to be eligible for analysis. During the PD procedure, the conventional PD dialysis fluids include $1.5 \%$ or $2.5 \%$ dextrose and the twin-bag system was applied for all PD patients. For the management of dyslipidemia in the patients, lipid-lowering agents were formulated and given to the patients $(10.3 \%)$. As shown in Fig. 1, the mean $( \pm$ SD) age of the subjects was $49.9 \pm 14.5$ years (57.6\% were men). $10.1 \%$ of patients had prior CVD and $19.3 \%$ of patients had diabetic history. The leading cause of ESRD was chronic glomerulonephritis, which accounted for $64.3 \%$ of the group, followed by diabetic nephropathy (16.3\%) and hypertension (12.8\%).

\section{Apo B/apo A1 tertiles}

The baseline of the apo B/apo A1 ratio ranged between 0.22 and 2.24 (interquartile range $=0.50-0.85 \mathrm{U} / \mathrm{L}$, mean $=0.65$ ) and the characteristics of the patients stratified by tertiles of the apo B/apo A1 ratio are displayed in Table 1. As shown in Table 1, patients with a higher apo B/apo A1 ratio presented with diabetic tendency, and increased $\mathrm{BMI}, \mathrm{tKt} / \mathrm{V}, \mathrm{eGFR}, \mathrm{N} / \mathrm{L}$, hemoglobin, TG, CHOL, LDL, apo B and Lp (a), and decreased levels of HDL and apo A1 $(P<0.05)$. No significant differences among age, sex, CVD, blood pressure, albumin, and lipidlowering agents use were observed (Table 1).

\section{The correlation of lipid parameters}

As shown in Table 2, the parameter apo A1 was strongly correlated with $\mathrm{HDL}$ and the apo B/apo A1 ratio $(r=0.60$ and -0.51 , respectively). Apo $B$ was strongly correlated with LDL and the ratio of apo B/apo A1 $(r=0.74$, and 0.81 , respectively). On the other hand, the ratio of $\mathrm{N} / \mathrm{L}$ was negatively correlated with apo A1 $(r=-0.10)$ but not with apo B $(r=0.04$; Table 2).

\section{The correlation of Apo B/apo A1, all-cause mortality, and cardiovascular events}

In this study, the median follow-up period was 27 months (interquartile range $=13-41$ months). By the end of the study, 202 patients $(23.5 \%)$ were died and recorded. Fifty-two patients $(6.0 \%)$ had received kidney transplantation, 127 patients $(14.8 \%)$ were transferred to HD, 6 patients $(0.7 \%)$ transferred to other PD centers, 20 patients $(2.3 \%)$ patients discontinued follow-up, and the remaining 453 patients (52.7\%) were still followed in our PD center. Of the 202 deaths, 108 were caused by CVD (53.5\%), 16 were caused by infectious disease ( 7 . $9 \%), 3$ were due to malignancy (1.5\%), 16 were caused by cachexia (7.9\%), and 19 and 40 cases (9.4 and 19.8\%, respectively) were caused by other and unknown reasons, respectively. The correlation between all-cause mortality and the apo B/apo A1 ratio was evaluated using Kaplan-Meier methods, which are shown in Fig. 2. The survival rate was also estimated at the end of 1,3 , and 5 years as follows: $94.9,80.7$, and $64.5 \%$, in the T1 group; 95.1, 73.7, and 58.3\%, in the T2 group; and 90.6, 72.2, and $52.5 \%$ in the T3 group. The results showed that the total survival rate in the T3 group was significantly lower

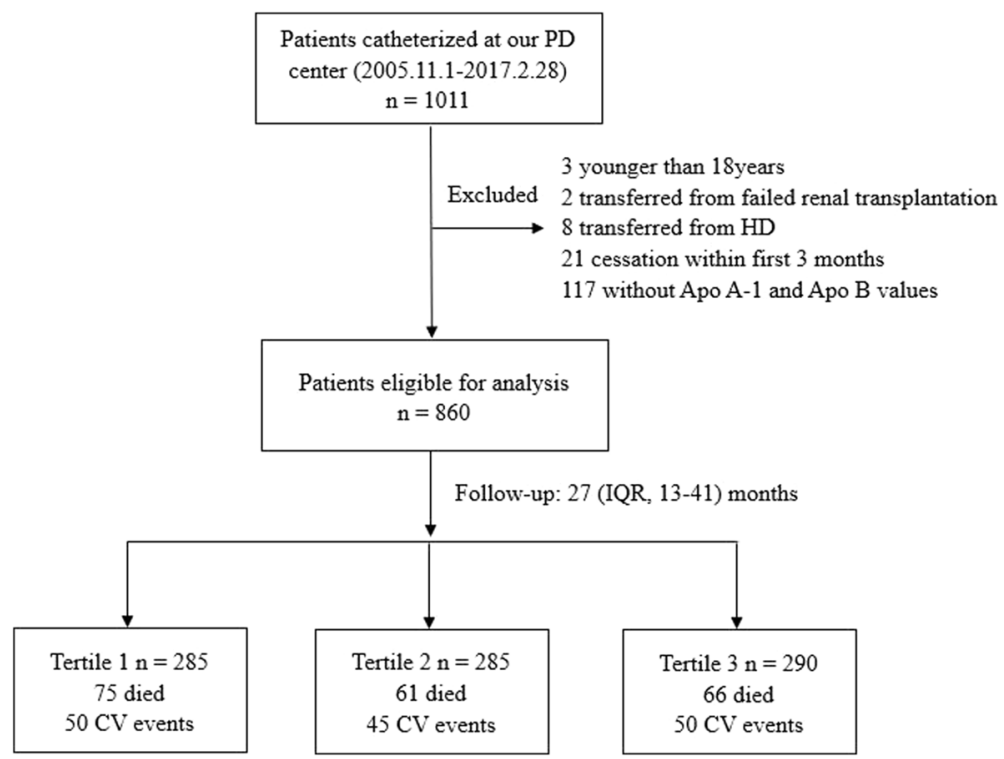

Fig. 1 Enrollment flow chart for analysis. PD, peritoneal dialysis; HD, hemodialysis; Apo A1, apolipoprotein A1;Apo B, apolipoprotein B; IQR, interquartile range; $\mathrm{CV}$, cardiovascular 
Table 1 Baseline characteristics of individuals stratified by tertiles of apo B/apo A1 ratio

\begin{tabular}{|c|c|c|c|c|c|}
\hline \multirow[t]{2}{*}{ Variables } & \multicolumn{3}{|c|}{ Apo B/Apo A1 Ratio Tertiles } & \multirow[t]{2}{*}{ Total } & \multirow[t]{2}{*}{$P$ Value } \\
\hline & $\leq 0.545(n=285)$ & $0.545-0.769(n=285)$ & $>0.769(n=290)$ & & \\
\hline Age $(y r)$ & $50.9 \pm 14.8$ & $48.7 \pm 14.3$ & $50.0 \pm 14.5$ & $49.9 \pm 14.5$ & 0.200 \\
\hline Men (\%) & $164(57.5)$ & $172(60.4)$ & $159(54.8)$ & $495(57.6)$ & 0.408 \\
\hline Body mass index $\left(\mathrm{kg} / \mathrm{m}^{2}\right)$ & $21.0 \pm 3.2$ & $22.2 \pm 3.5$ & $22.6 \pm 3.4$ & $21.9 \pm 3.4$ & $<0.001$ \\
\hline Diabetes (\%) & $46(16.1)$ & $50(17.5)$ & $70(24.1)$ & $166(19.3)$ & 0.034 \\
\hline CVD (\%) & $25(8.8)$ & $25(8.8)$ & $37(12.8)$ & $87(10.1)$ & 0.186 \\
\hline Systolic pressure (mmHg) & $145 \pm 27$ & $148 \pm 26$ & $147 \pm 26$ & $147 \pm 26$ & 0.541 \\
\hline Diastolic pressure $(\mathrm{mmHg})$ & $87 \pm 17$ & $90 \pm 15$ & $88 \pm 15$ & $87 \pm 17$ & 0.067 \\
\hline Total Kt/N & $2.01(1.43,2.53)$ & $2.26(1.79,2.69)$ & $2.23(1.81,2.80)$ & $2.18(1.68,2.70)$ & $<0.001$ \\
\hline eGFR (ml/min per $\left.1.73 \mathrm{~m}^{2}\right)$ & $2.66(1.54,4.09)$ & $3.59(2.02,5.82)$ & $3.85(1.89,6.07)$ & $3.26(1.78,5.56)$ & $<0.001$ \\
\hline Hemoglobin (g/L) & $76.65 \pm 16.48$ & $79.71 \pm 16.19$ & $80.74 \pm 16.75$ & $79.04 \pm 16.55$ & 0.009 \\
\hline $\mathrm{N} / \mathrm{L}$ & $3.27(2.37,4.75)$ & $3.50(2.53,5.53)$ & $3.86(2.61,5.20)$ & $3.58(2.50,5.12)$ & 0.028 \\
\hline Albumin (g/L) & $35.90 \pm 5.00$ & $35.70 \pm 5.31$ & $34.50 \pm 5.29$ & $35.36 \pm 5.23$ & 0.848 \\
\hline Total cholesterol (mmol/L) & $3.56(3.05,4.30)$ & $4.03(3.46,4.80)$ & $4.55(3.84,5.40)$ & $4.06(3.34,4.89)$ & $<0.001$ \\
\hline Triglyceride (mmol/L) & $1.00(0.73,1.36)$ & $1.27(0.91,1.72)$ & $1.68(1.14,2.21)$ & $1.28(0.89,1.78)$ & $<0.001$ \\
\hline Low density lipoprotein (mmol/L) & $1.90(1.40,2.30)$ & $2.31(1.89,2.93)$ & $2.81(2.24,3.46)$ & $2.30(1.83,2.94)$ & $<0.001$ \\
\hline High density lipoprotein (mmol/L) & $1.20(0.96,1.53)$ & $1.14(0.92,1.42)$ & $0.98(0.81,1.16)$ & $1.08(0.89,1.37)$ & $<0.001$ \\
\hline Apo B (g/L) & $0.60(0.50,0.70)$ & $0.80(0.70,0.90)$ & $1.07(0.90,1.41)$ & $0.80(0.63,1.00)$ & $<0.001$ \\
\hline Apo A1 (g/L) & $1.38(1.22,1.59)$ & $1.23(1.10,1.40)$ & $1.09(0.98,1.23)$ & $1.22(1.07,1.41)$ & $<0.001$ \\
\hline Lipoprotein (a) (mg/L) & $284(141,514)$ & $303(160,501)$ & $409(226,663)$ & $328(172,586)$ & $<0.001$ \\
\hline Lipid-lowering agents use (\%) & $29(10.2)$ & $36(12.6)$ & $24(8.3)$ & $89(10.3)$ & 0.228 \\
\hline
\end{tabular}

CVD cardiovascular disease, $N / L$ neutrophil to lymphocyte ratio, $A p o$, apolipoprotein $B, A p o A 1$ apolipoprotein $A 1$

$P<0.05$ is considered statistically significant

compared to the $\mathrm{T} 1$ and $\mathrm{T} 2$ groups $(P=0.017)$ (Fig. 2a). The cardiovascular events-free survival rate at the end of 1,3 , and 5 years was (respectively) $96.0,85.8$, and $72.8 \%$ in the T1 group; $94.3,79.2$, and $70.2 \%$ in the T2 group; and $90.4,78.8$, and $61.0 \%$ in the T3 group. Similarly, the cardiovascular events-free survival rate in the T3 group was lowest among these groups $(P=0.021)$ (Fig. $2 b)$. The association between the apo $\mathrm{B} / \mathrm{apo} \mathrm{A} 1$ ratio and all-cause mortality and cardiovascular events was determined using
Cox regression analysis. As shown in Table 3, despite the adjustment in models 2 and 3, the apo B/apo A1 ratio was still associated with all-cause mortality and cardiovascular events. In model 3, the HRs and 95\% CIs for tertile 3 versus tertile 1 were: HR, 1.60 (95\% CI: 1.02 to 2.49), and HR, 2.04 (95\% CI: 1.21 to 3.44), for all-cause mortality and cardiovascular events, respectively. These results suggest that apo A1 was correlated with all-cause mortality in models 2 and 3 , and cardiovascular events in models 1

Table 2 Correlation between Apo B/Apo A1 and parameters of lipid and inflammation

\begin{tabular}{|c|c|c|c|c|c|c|c|c|}
\hline & Apo B/Apo A1 & $\mathrm{CHOL}$ & TG & LDL & $\mathrm{HDL}$ & Apo A1 & Apo B & $\operatorname{Lp}(\mathrm{a})$ \\
\hline $\mathrm{CHOL}$ & $0.40^{\mathrm{a}}$ & & & & & & & \\
\hline TG & $0.45^{\mathrm{a}}$ & $0.38^{\mathrm{a}}$ & & & & & & \\
\hline LDL & $0.51^{\mathrm{a}}$ & $0.88^{\mathrm{a}}$ & $0.32^{\mathrm{a}}$ & & & & & \\
\hline $\mathrm{HDL}$ & $-0.29^{\mathrm{a}}$ & $0.43^{\mathrm{a}}$ & $-0.30^{a}$ & $0.28^{a}$ & & & & \\
\hline Apo A1 & $-0.51^{\mathrm{a}}$ & $0.35^{\mathrm{a}}$ & $-0.09^{\mathrm{b}}$ & $0.20^{\mathrm{a}}$ & $0.60^{\mathrm{a}}$ & & & \\
\hline Apo B & $0.81^{\mathrm{a}}$ & $0.72^{\mathrm{a}}$ & $0.46^{\mathrm{a}}$ & $0.74^{\mathrm{a}}$ & $0.05^{c}$ & $0.03^{c}$ & & \\
\hline Lp (a) & $0.16^{\mathrm{a}}$ & $0.27^{\mathrm{a}}$ & $0.03^{c}$ & $0.28^{\mathrm{a}}$ & $0.08^{b}$ & $0.09^{\mathrm{a}}$ & $0.25^{\mathrm{a}}$ & \\
\hline N/L & $0.08^{\mathrm{b}}$ & $-0.00^{c}$ & $-0.01^{c}$ & $-0.01^{c}$ & $-0.02^{c}$ & $-0.10^{\mathrm{a}}$ & $0.04^{c}$ & $0.03^{c}$ \\
\hline
\end{tabular}

CHOL cholesterol, $T G$ triglyceride, $L D L$ low density lipoprotein, $H D L$ high density lipoprotein, $A p o A 1$ apolipoprotein $A 1, A p o B$ apolipoprotein $B, L p(a)$ lipoprotein (a), $N / L$ neutrophil to lymphocyte ratio

${ }^{a}$ Correlation is significant at the 0.01 level (two-tailed)

${ }^{\mathrm{b} C}$ Correlation is significant at the 0.05 level (two-tailed)

${ }^{\mathrm{c} C}$ Correlation is not significant 


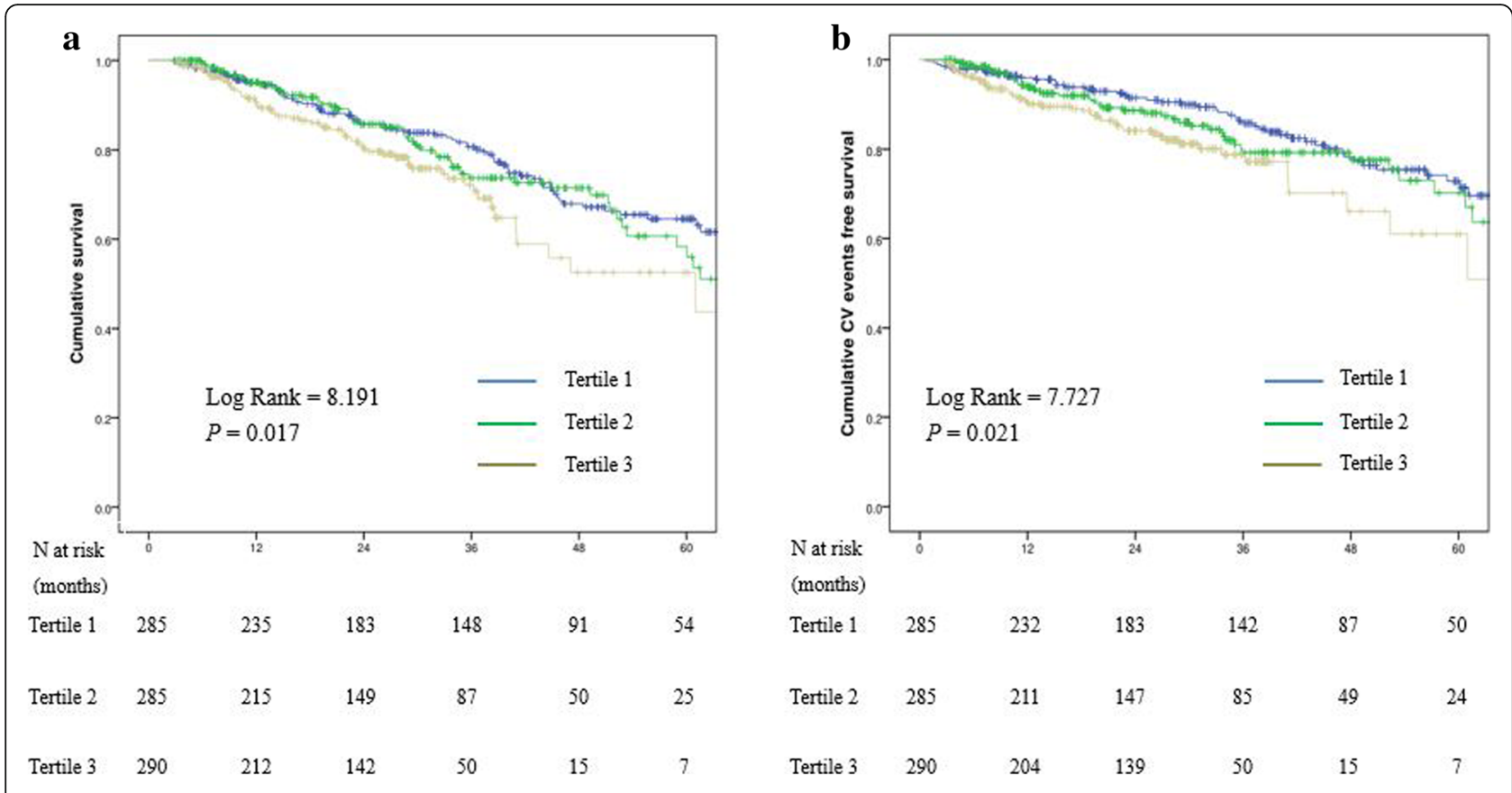

Fig. 2 Survival curves for patients stratified by Apo B/Apo A1 ratio. a All-cause mortality-free survival curves. b Cardiovascular events-free survival curves

and 2. Apo B was not correlated with either parameter in models 1-3 (Table 3).

\section{Discussion}

In this retrospective cohort study, we identified the correlation between the serum apo $\mathrm{B} / \mathrm{apo} \mathrm{A} 1$ ratio and PD patient characteristics. We found, for the first time, that a higher apo B/apo A1 ratio is strongly correlated with increased all-cause mortality and cardiovascular events in PD patients. Consistent with the empirical results from HD patients and non-CKD patients $[9,12-15]$, the apo $\mathrm{B} / \mathrm{apo} \mathrm{A} 1$ ratio can be used as an important risk indicator for clinicians in various fields, including dialysis.

Apo B is a key basal unit of atherogenic lipoprotein including VLDL, IDL, and LDL. Since each of these lipoproteins detects only one apo B molecule, apo B measurements have been used to determine the precise number of the atherogenic lipoproteins in patients [7]. Previous studies indicated that apo B was a strong predictor of mortality and cardiovascular risk $[9,16]$. Contrary to these studies, we did not find a significant correlation between apo B and these events. Some differences between these studies and our results are worth

Table 3 The associations of apo A1, apo B, and the apo B/apo A1 ratio (Tertile3 Vs Tertile1) with all-cause mortality and cardiovascular events

\begin{tabular}{|c|c|c|c|c|c|c|}
\hline \multirow[t]{2}{*}{ Variables } & \multicolumn{2}{|l|}{ Apo A1 } & \multicolumn{2}{|l|}{ Apo B } & \multicolumn{2}{|c|}{ Apo B/Apo A1 ratio ${ }^{d}$} \\
\hline & HR $(95 \%$ Cl) & $P$ Value & $\mathrm{HR}(95 \% \mathrm{Cl})$ & $P$ Value & HR (95\% Cl) & $P$ Value \\
\hline \multicolumn{7}{|c|}{ All-cause mortality } \\
\hline Model $1^{a}$ & $0.64(0.39-1.08)$ & 0.094 & $1.22(0.78-1.90)$ & 0.384 & $1.62(1.15-2.29)$ & 0.005 \\
\hline Model $2^{\mathrm{b}}$ & $0.47(0.25-0.89)$ & 0.020 & $0.92(0.52-1.65)$ & 0.789 & $1.59(1.05-2.43)$ & 0.031 \\
\hline Model $3^{c}$ & $0.48(0.24-0.94)$ & 0.033 & $0.73(0.31-1.72)$ & 0.466 & $1.60(1.02-2.49)$ & 0.040 \\
\hline \multicolumn{7}{|c|}{ Cardiovascular events } \\
\hline Model $1^{\mathrm{a}}$ & $0.47(0.25-0.90)$ & 0.022 & $1.04(0.60-1.80)$ & 0.900 & $1.76(1.17-2.63)$ & 0.006 \\
\hline Model $2^{\mathrm{b}}$ & $0.39(0.18-0.83)$ & 0.015 & $0.80(0.40-1.61)$ & 0.535 & $1.72(1.05-2.81)$ & 0.030 \\
\hline Model $3^{c}$ & $0.44(0.19-1.02)$ & 0.055 & $1.14(0.42-3.10)$ & 0.795 & $2.04(1.21-3.44)$ & 0.008 \\
\hline
\end{tabular}

Apo A-1 apolipoprotein A-1, Apo B apolipoprotein B, HR Hazard ratios, 95\% Cl 95\% confidence intervals

${ }^{\mathrm{b}}$ Model 2: adjusted for age, sex, BMI, diabetes, CVD, SBP, eGFR, tKt/N, hemoglobin, N/L, and albumin

'Model 3: model 2 adjusted for cholesterol, triglyceride, and lipid-lowering agents use

${ }^{\mathrm{d}}$ Tertile 3 versus tertile 1 
noting. First, in our cohort study, the follow-up time was approximately 2 years, whereas other studies have follow-up times of more than 7 years. Second, we enrolled incident PD patients in our study, whereas Sato et al. [9] assessed the changes apo B in prevalent HD patients. These possibilities may cause the variations between our results and others, and these contradictions should be further elucidated in future studies.

In contrast to apo B, apo A1 is a main constituent of HDL. Although each HDL particle contains five apo A1 molecules, systemic apo A1 levels have been used as an indicator for HDL cholesterol concentrations [17]. The relationship between apo A1 and cardiovascular events remains controversial. A large meta-analysis of prospective studies in the general populations shows an inverse association between apo A1 and incident coronary heart disease [18]. However, Zhu et al. [16] found that apo A1 was not correlated with cardiovascular events in the general population. In the diabetes dialysis study in German, apo A1 failed to predict cardiovascular events or all-cause mortality [19]. Sato et al. [9] also found that apo A1 was not independently associated with CVDrelated and all-cause mortality. Interesting, in this study, we found that apo A1 had a higher association with the prevention of mortality in adjusted models, and cardiovascular events in model 1 and model 2 , with a marginal significance for cardiovascular events in model 3. We speculate that the difference in population and follow-up time may be important factors for apo A1 adjustment and application.

The apo B/apo A1 ratio is a typical marker for the atherogenic /antiatherogenic condition, and a higher apo $\mathrm{B} / \mathrm{apo} \mathrm{A} 1$ ratio represents progression in the atherosclerotic state. Previous studies have suggested that a high apo B/apo A1 ratio strongly correlates with pathophysiological markers of atherosclerosis defined by coronary angiography $[8,20]$, and calcium scores of the coronary arteries [21], and by ultrasound techniques such as the thickness of carotid arteries [16, 22, 23], endothelial dysfunction [24] and existence of femoral plaques [25]. In addition, a high apo B/apo A1 ratio can also be used to predict risk of carotid plaque development [26] and major adverse cardiovascular events [14, 27-29]. Our findings are in agreement with these studies and show that the apo B/apo A1 ratio is independently associated with cardiovascular events.

Although the apo B/apo A1 ratio is considered as a risk predictor for CVD mortality in the general population $[14,30]$, to date, few studies have used the apo B/ apo A1 ratio to predict the mortality of dialysis patients. The results were also conflicting. For example, Sato et al. [9] showed that a higher apo B/apo A1 ratio was associated with an increased risk of all-cause and CVDrelated mortality in prevalent HD patients. However, in that study, BMI, which has been associated lower mortality risk, was not adjusted. Another study demonstrated that the baseline apo B/apo A1 ratio did not correlate with 4-year mortality [31]. In that study, after adjustment for confounding factors, a higher apo B/apo A1 ratio predicted first-year survival and the next 3 years mortality, and an increased ratio during the first year was also associated with a survival advantage in dialysis patients. However, this association lost significance after adjustment for the protein-energy wasting index. In this study, our findings extended this observation and indicated that the increased risk of all-cause mortality is associated with an increase of the apo B/apo A1 ratio, independent of BMI and N/L, in PD patients. It was reported that the lipid profile was different in PD and $\mathrm{HD}$, and the apo B/apo A1 ratio was higher in patients on HD than patients receiving PD [32], which is supported by the previous study by Sato et al. [9] and our results. However, our study indicated that the association between the apo B/apo A1 ratio and mortality in PD patients was similar to the previous study in HD patients [9].

The present study also has limitations to discuss. First, this is a retrospective study, which can only lead to associations but not causality. Second, the number of patients is relatively small. Third, in this study, we only applied the baseline data but did not exclude patients who had already taken lipid-lowering medications, nor considered the data changes during the follow-up period. Fourth, because of the limited sample size, the potential risk factors were not all adjusted in this cohort study. Hence, the effects of residual confounding factors cannot be eliminated completely. Our future studies will address the improvement of these issues.

\section{Conclusions}

In summary, despite conflicting evidence of lipid effects on the prognosis of PD patients, our results found significant associations between the apo B/apo A1 ratio, cardiovascular events, and all-cause mortality in $\mathrm{PD}$ patients. The apo B/apo A1 ratio can serve as a risk marker for the risk of the CVDs and mortality in PD patients.

\section{Availability of data and materials \\ All data generated or analyzed during this study are included in this published article.}

\section{Authors' contributions \\ $X Z$ conceived the study, YC, CY and SL participated in the design, $L D, Y Y, P Q$, DP, BZ collected the data, XZ performed statistical analyses, XZ drafted the manuscript, QC edited and checked the manuscript. All of the authors have read and approved the final manuscript.}

Ethics approval and consent to participate

The study was approved by the ethics committee of The First Affiliated Hospital of Nanchang University. It was conducted according to the standards of the Declaration of Helsinki. 


\section{Competing interests}

The authors declare that they have no competing interests.

\section{Publisher's Note}

Springer Nature remains neutral with regard to jurisdictional claims in published maps and institutional affiliations.

\section{Received: 17 March 2018 Accepted: 4 May 2018 Published online: 17 May 2018}

\section{References}

1. Kaysen GA. Hyperlipidemia of chronic renal failure. Blood Purif. 1994;12:60-7.

2. Ikewaki K. In vivo kinetic studies to further understand pathogenesis of abnormal lipoprotein metabolism in chronic kidney disease. Clin Exp Nephrol. 2014;18:261-4.

3. Zhao W, Zheng XL, Jiang ZN, Liao XB, Zhao SP. Risk factors associated with atherogenic dyslipidemia in the presence of optimal statin therapy. Int $J$ Cardiol. 2017;248:355-60

4. Quispe R, Manalac RJ, Faridi KF, Blaha MJ, Toth PP, Kulkarni KR, Nasir K, Virani SS, Banach M, Blumenthal RS, Martin SS, Jones SR. Relationship of the triglyceride to high-density lipoprotein cholesterol (TG/HDL-C) ratio to the remainder of the lipid profile: the very large database of Lipids-4 (VLDL-4) study. Atherosclerosis. 2015;242:243-50.

5. Yusuf S, Hawken S, Ounpuu S, Dans T, Avezum A, Lanas F, McQueen M, Budaj A, Pais P, Varigos J, Lisheng L. Effect of potentially modifiable risk factors associated with myocardial infarction in 52 countries (the INTERHEART study): case-control study. Lancet. 2004;364:937-52.

6. Sniderman AD, Pedersen T, Kjekshus J. Putting low-density lipoproteins at center stage in atherogenesis. Am J Cardiol. 1997;79:64-7.

7. Andrikoula M, McDowell IF. The contribution of $A p o B$ and $A p o A 1$ measurements to cardiovascular risk assessment. Diabetes Obes Metab. 2008;10:271-8.

8. Song Y, Yang Y, Zhang J, Wang Y, He W, Zhang X, Zhu J, Lu Z. The apoB100/apoAl ratio is independently associated with the severity of coronary heart disease: a cross sectional study in patients undergoing coronary angiography. Lipids Health Dis. 2015;14:150.

9. Sato Y, Fujimoto S, Toida T, Nakagawa H, Yamashita Y, Iwakiri T, Fukuda A, Iwatsubo S. Apoprotein B/Apoprotein a-1 ratio and mortality among prevalent dialysis patients. Clin J Am Soc Nephrol. 2016;11:840-6.

10. Samouilidou EC, Karpouza AP, Kostopoulos V, Bakirtzi T, Pantelias K, Petras D, Tzanatou-Exarchou H, GE J. Lipid abnormalities and oxidized LDL in chronic kidney disease patients on hemodialysis and peritoneal dialysis. Ren Fail. 2012;34:160-4

11. Wheeler DC, London GM, Parfrey PS, Block GA, Correa-Rotter R, Dehmel B, Drueke TB, Floege J, Kubo Y, Mahaffey KW, Goodman WG, Moe SM, Trotman ML, Abdalla S, Chertow GM, Herzog CA. Effects of cinacalcet on atherosclerotic and nonatherosclerotic cardiovascular events in patients receiving hemodialysis: the EValuation of Cinacalcet $\mathrm{HCl}$ therapy to lower CardioVascular events (EVOLVE) trial. J Am Heart Assoc. 2014;3:e1363.

12. Walldius G, Jungner I, Holme I, Aastveit AH, Kolar W, Steiner E. High apolipoprotein B, low apolipoprotein A-I, and improvement in the prediction of fatal myocardial infarction (AMORIS study): a prospective study. Lancet. 2001;358:2026-33.

13. Dunder $K$, Lind L, Zethelius B, Berglund L, Lithell $H$. Evaluation of a scoring scheme, including proinsulin and the apolipoprotein B/apolipoprotein A1 ratio, for the risk of acute coronary events in middle-aged men: Uppsala longitudinal study of adult men (ULSAM). Am Heart J. 2004;148:596-601.

14. Walldius G, Aastveit AH, Jungner I. Stroke mortality and the apoB/apoA-I ratio: results of the AMORIS prospective study. J Intern Med. 2006;259:259-66.

15. Walldius $G$, Jungner I. The apoB/apoA-I ratio: a strong, new risk factor for cardiovascular disease and a target for lipid-lowering therapy-a review of the evidence. J Intern Med. 2006;259:493-519.

16. Zhu YM, Verma S, Fung M, McQueen MJ, Anderson TJ, Lonn EM. Association of apolipoproteins $b$ and $a-1$ with markers of vascular health or cardiovascular events. Can J Cardiol. 2017;33:1305-11.

17. Catapano AL, Graham I, De Backer G, Wiklund O, Chapman MJ, Drexel H, Hoes AW, Jennings CS, Landmesser U, Pedersen TR, Reiner Z, Riccardi G, Taskinen MR, Tokgozoglu L, Verschuren WM, Vlachopoulos C, Wood DA, Zamorano JL. 2016 ESC/EAS guidelines for the Management of Dyslipidaemias. Eur Heart J. 2016;37:2999-3058.
18. Thompson A, Danesh J. Associations between apolipoprotein B, apolipoprotein $\mathrm{Al}$, the apolipoprotein B/Al ratio and coronary heart disease: a literature-based meta-analysis of prospective studies. J Intern Med. 2006;259:481-92.

19. Silbernagel G, Genser B, Drechsler C, Scharnagl H, Grammer TB, Stojakovic T, Krane V, Ritz E, Wanner C, Marz W. HDL cholesterol, apolipoproteins, and cardiovascular risk in hemodialysis patients. J Am Soc Nephrol. 2015;26:484-92.

20. Liting P, Guoping L, Zhenyue C. Apolipoprotein B/apolipoprotein A1 ratio and non-high-density lipoprotein cholesterol. Predictive value for CHD severity and prognostic utility in CHD patients. Herz. 2015;40(Suppl 1):1-7.

21. Simon A, Chironi G, Gariepy J, Del PM, Levenson J. Differences between markers of atherogenic lipoproteins in predicting high cardiovascular risk and subclinical atherosclerosis in asymptomatic men. Atherosclerosis. 2005:179:339-44.

22. Huang F, Yang Z, Xu B, Bi Y, Xu M, Xu Y, Lu J, Liu Y, Dai M, Zhou W, Wang W, Chen Y. Both serum apolipoprotein B and the apolipoprotein B/ apolipoprotein A-I ratio are associated with carotid intima-media thickness. PLoS One. 2013;8:e54628.

23. Dahlen EM, Lanne T, Engvall J, Lindstrom T, Grodzinsky E, Nystrom FH, Ostgren CJ. Carotid intima-media thickness and apolipoprotein B/ apolipoprotein A-I ratio in middle-aged patients with type 2 diabetes. Diabet Med. 2009;26:384-90.

24. Emoto T, Sawada T, Morimoto N, Tenjin T, Wakimoto T, Ikeda F, Sato C, Terashita D, Mizoguchi T, Mizuguchi T, Okamoto H, Matsuo Y, Kim SK, Takarada A, Yokoyama M. The apolipoprotein B/A1 ratio is associated with reactive oxygen metabolites and endothelial dysfunction in statin-treated patients with coronary artery disease. J Atheroscler Thromb. 2013;20:623-9.

25. Schmidt C, Fagerberg B. ApoB/apoA-I ratio is related to femoral artery plaques in 64-year-old women also in cases with low LDL cholesterol. Atherosclerosis. 2008;196:817-22.

26. Steffen BT, Guan W, Remaley AT, Stein JH, Tattersall MC, Kaufman J, Tsai MY. Apolipoprotein $B$ is associated with carotid atherosclerosis progression independent of individual cholesterol measures in a 9-year prospective study of multi-ethnic study of atherosclerosis participants. J Clin Lipidol. 2017;11:1181-91.

27. van der Steeg WA, Boekholdt SM, Stein EA, El-Harchaoui K, Stroes ES, Sandhu MS, Wareham NJ, Jukema JW, Luben R, Zwinderman AH, Kastelein $\mathrm{J}$, Khaw KT. Role of the apolipoprotein B-apolipoprotein A-I ratio in cardiovascular risk assessment: a case-control analysis in EPIC-Norfolk. Ann Intern Med. 2007;146:640-8.

28. Albers JJ, Slee A, O'Brien KD, Robinson JG, Kashyap ML, Kwiterovich PJ, Xu P Marcovina SM. Relationship of apolipoproteins A-1 and B, and lipoprotein(a) to cardiovascular outcomes: the AIM-HIGH trial (Atherothrombosis intervention in metabolic syndrome with low HDL/high triglyceride and impact on Global Health outcomes). J Am Coll Cardiol. 2013;62:1575-9.

29. Kappelle PJ, Gansevoort RT, Hillege JL, Wolffenbuttel BH, Dullaart RP. Apolipoprotein B/A-I and total cholesterol/high-density lipoprotein cholesterol ratios both predict cardiovascular events in the general population independently of nonlipid risk factors, albuminuria and Creactive protein. J Intern Med. 2011;269:232-42.

30. Sierra-Johnson J, Fisher RM, Romero-Corral A, Somers VK, Lopez-Jimenez F, Ohrvik J, Walldius G, Hellenius ML, Hamsten A. Concentration of apolipoprotein B is comparable with the apolipoprotein B/apolipoprotein AI ratio and better than routine clinical lipid measurements in predicting coronary heart disease mortality: findings from a multi-ethnic US population. Eur Heart J. 2009;30:710-7.

31. Chmielewski M, Carrero JJ, Qureshi AR, Axelsson J, Heimburger O, Berglund $L$, Barany P, Rutkowski B, Lindholm B, Stenvinkel P. Temporal discrepancies in the association between the apoB/apoA-I ratio and mortality in incident dialysis patients. J Intern Med. 2009:265:708-16.

32. Liu J, Rosner MH. Lipid abnormalities associated with end-stage renal disease. Semin Dial. 2006:19:32-40. 